\title{
Comparison of IVF-ET outcomes in patients with hydrosalpinx pretreated with either sclerotherapy or laparoscopic salpingectomy
}

\author{
Eun Duc $\mathrm{Na}^{1}$, Dong Hyun Cha', Jung Hyun $\mathrm{Cho}^{2}$, Mi Kyoung Kim² \\ Departments of 'Obstetrics and Gynecology and '2Reproductive Medicine, CHA Gangnam Medical Center, CHA University, Seoul, Korea
}

Objective: Many studies have demonstrated that hydrosalpinx has a detrimental effect on the outcome of IVF. Treating hydrosalpinges prior to the IVF procedure in women with hydrosalpinges is thought to improve the likelihood of successful IVF outcome. Vaginal ultrasound-guided aspiration of hydrosalpinx fluid (HSF) with injection of the sclerosing agent in situ might be simpler than invasive procedures like salpingectomy. Therefore, we carried out a retrospective study on the effects of ultrasound-guided HSF aspiration and injection of the sclerosing agent of ultrasonically diagnosed hydrosalpinx on IVF outcome.

Methods: In our retrospective study, 97 tubal factor infertile female patients that underwent IVF treatment between January 2005 and December 2012 at the Reproductive Medicine Center of CHA Hospital were divided into two study groups. Fifty-six patients underwent interventional ultrasound sclerotherapy (group 1), and the remaining 41 patients received laparoscopic salpingectomy (group 2) before IVF. We compared the IVF outcomes of the two groups.

Results: The results showed that ultrasound-guided HSF aspiration and sclerotherapy have IVF outcomes comparable to laparoscopic salpingectomy.

Conclusion: Interventional ultrasound guided sclerotherapy before IVF is an effective and less invasive prophylactic intervention alternative to salpingectomy with hydrosalpinx.

Keywords: Hydrosalpinx; IVF; Salpingectomy

\section{Introduction}

Hydrosalpinx refers to a pathologic condition in which distally obstructed salpinges of various pathologies become filled with fluid, forming a saccular structure. The prevalence of this disease is known to be up to $30 \%$ in women with tubal pathology [1,2]. Hysterosalpingography and ultrasound imaging are relatively simple and precise tests for the diagnosis of hydrosalpinx, and diagnosis can be confirm-

Received: Oct 19, 2012 · Revised: Nov 9, 2012 · Accepted: Nov 23, 2012 Corresponding author: Jung Hyun Cho

Department of Reproductive Medicine, CHA Gangnam Medical Center, CHA University, 566 Nonhyeon-ro, Gangnam-gu, Seoul 135-913, Korea Tel: +82-2-3468-3410 Fax:+82-2-3468-3449 E-mail:medicho@hotmail.com

This is an Open Access article distributed under the terms of the Creative Commons Attribution Non-Commercial License (http://creativecommons.org/licenses/by-nc/3.0/) which permits unrestricted non-commercial use, distribution, and reproduction in any medium, provided the original work is properly cited. ed by visual inspection during operation [3].

In many studies, hydrosalpinx has been associated with unfavorable IVF outcomes and reduced implantation. The presence of hydrosalpinx fluid in the endometrial cavity physically inhibits implantation and impairs the expression of factors essential for the development and differentiation of the endometrium, such as $\beta$-integrin, leukemia inhibitory factor, and HOXA10 mRNA [4,5]. Hydrosalpinx acts directly on the embryos and the endometrium, impairing endometrial receptivity; reduces the IVF success rate, implantation rate, and pregnancy rate by 50 percent; and doubles the spontaneous abortion rate [6,7].

Up to the present, surgical options for women with hydrosalpinges have been either resection of hydrosalpinx or salpingoplasty. Resection of hydrosalpinges may reduce the blood flow to the ovaries, resulting in a reduced number of retrieved oocytes in stimulated cycles 
$[8,9]$. Salpingoplasty is associated with high recurrence rates and may result in chronic accumulation of fluid that interferes with implantation. These procedures are usually performed by laparoscopy that requires hospitalization and general anesthesia. Patients may suffer from postoperative pain, discomfort, and inconvenience in everyday life.

We inserted a long needle and placed the tip inside the hydrosalpinx for aspiration, alcohol washing, and injection of tetracycline. Sclerotherapy hardens the salpingeal wall and also reduces secretion. We analyzed the effect of this procedure on pregnancy rates in IVF cycles.

Sclerotherapy can stop substances that inhibit implantation from formation and flow into the endometrial cavity. We investigated whether this procedure can improve the pregnancy rate, and at the same time, reduce the ectopic pregnancy rate. We compared the results of this procedure with the outcomes of laparoscopic surgery, hoping to discover the benefits of avoiding invasive procedures or hospitalization. Patients that underwent IVF procedures after removal of hydrosalpinx laparoscopically served as controls.

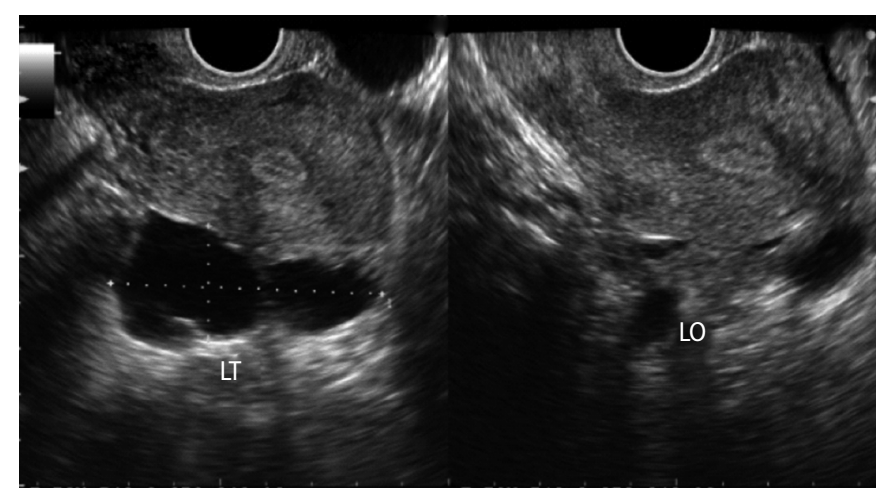

Figure 1. Transvaginal ultrasonogram of the patient with left hydrosalpinx before sclerotherapy. LT, left tube; LO, left ovary.

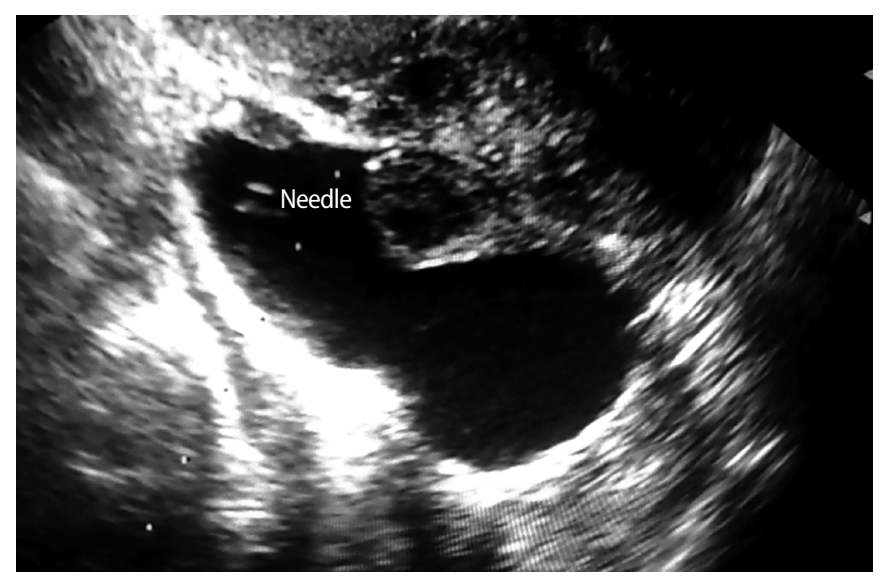

Figure 2. Needle in the middle of the left hydrosalpinx to aspirate hydrosalpinx fluid.

\section{Methods}

We retrospectively reviewed medical records of women who visited our fertility center and were diagnosed with infertility due to hydrosalpinx between January 2005 and December 2011. Patients with hydrosalpinx were classified into two groups according to the treatment they received. Group 1 consisted of patients treated with ultrasound-guided hydrosalpinx aspiration and sclerotherapy, and group 2 , of patients that underwent salpingectomy and served as controls. Patients in both groups received IVF therapy afterwards. In group 1, ultrasound-guided hydrosalpinx aspiration was performed under mild sedation. Following identification of hydrosalpinx via transvaginal probe (Figure 1), a 19 gauge Chiba needle (Aniomed Gmbh Medizintechnik KG, Karlsruhe, Germany) was carefully inserted into the widest part of the hydrosalpinx for aspiration. After aspiration of all the fluid (Figure 2), the tube cavity was washed with 95\% alcohol 5 to 6 times (Figure 3), and 5\% tetracycline (2 to $6 \mathrm{~mL}$ ) was then infused (Figure 4). In cases with bilateral disease, in order to prevent contamination between salpinges, another new needle was used for

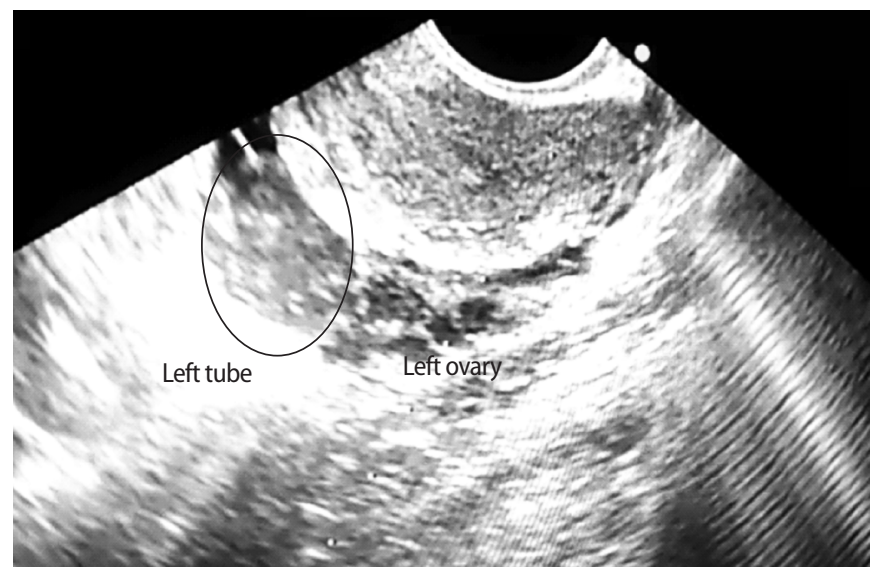

Figure 3. Irrigation tube with alcohol after aspiration of hydrosalpinx fluid.

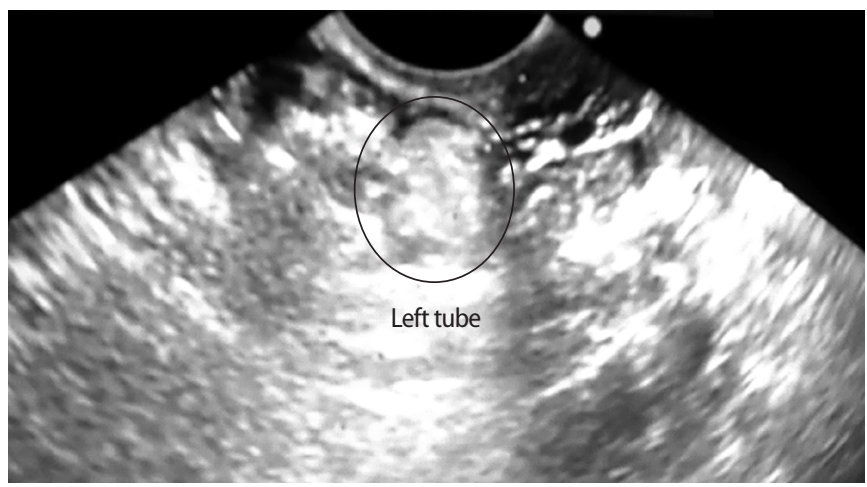

Figure 4. Injection of tetracycline emulsion into the tube after irrigation. 
the hydrosalpinx on the other side. If persistent hydrosalpinx was found on ultrasound imaging two weeks after intervention, this procedure was repeated two or three times (Figure 5). Oral antibiotics were prescribed for 3 days to prevent infection following sclerotherapy.

Between the two groups, we compared the following variables: age, duration of infertility, day 3 FSH levels, past medical and surgical history, number of oocytes retrieved after stimulation cycle, and pregnancy rate after IVF, with statistical analysis using Pearson's correlation and ANOVA.

\section{Results}

Fifty-six patients underwent sclerotherapy following aspiration (group 1), and 41 patients underwent laparoscopic salpingectomy (group 2). There were no statistical differences with respect to mean age (32.9 years vs. 32.4 years), duration of infertility (2.9 years vs. 3.8 years), body mass index (BMl, $21.5 \mathrm{~kg} / \mathrm{m}^{2} \mathrm{vs} .22 .2 \mathrm{~kg} / \mathrm{m}^{2}$ ), or day 3

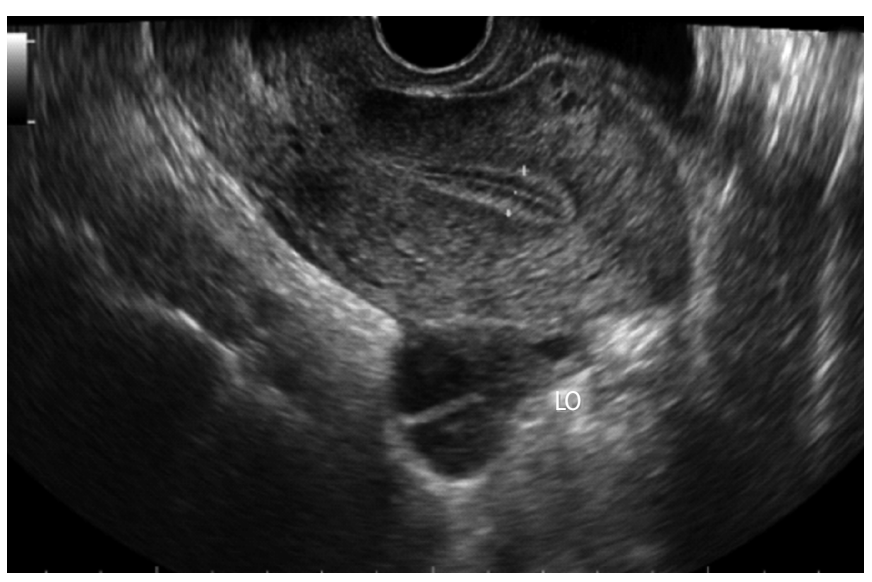

Figure 5. Transvaginal ultrasonogram of the patient who underwent sclerotherapy to the left hydrosalpinx 2 weeks earlier (same patient as Figure 1). LO, left ovary.

Table 1. Patient's characteristics and IVF outcomes in the two groups

\begin{tabular}{lccc}
\hline Variable & $\begin{array}{c}\text { Group 1 } \\
\text { (sclerotherapy) }\end{array}$ & $\begin{array}{c}\text { Group 2 } \\
\text { (salpingectomy) }\end{array}$ & $p$-value \\
\hline Patients & 56 & 41 & $\mathrm{NS}$ \\
Age & $32.9 \pm 4.1$ & $32.4 \pm 4.5$ & $\mathrm{NS}$ \\
Duration of infertility $(\mathrm{yr})$ & $2.9 \pm 1.8$ & $3.8 \pm 3.4$ & $\mathrm{NS}$ \\
Body mass index $\left(\mathrm{kg} / \mathrm{m}^{2}\right)$ & $21.5 \pm 2.2$ & $22.2 \pm 5.0$ & $\mathrm{NS}$ \\
Day 3 FSH & $10.9 \pm 17.1$ & $8.2 \pm 4.6$ & $\mathrm{NS}$ \\
IVF cycle & 60 & 46 & $\mathrm{NS}$ \\
Oocytes retrieved & $12.1 \pm 11$ & $6.2 \pm 1.0$ & $\mathrm{NS}$ \\
Clinical pregnancy rate & $23 / 60(38 \%)$ & $17 / 43(40 \%)$ & $\mathrm{NS}$ \\
Ectopic pregnancy rate & $2 / 56(3.6 \%)$ & $0 / 41(0 \%)$ & $\mathrm{NS}$ \\
Previous surgical history & $29 / 56(52 \%)$ & $12 / 41(29 \%)$ & 0.027 \\
\hline
\end{tabular}

Values are presented as mean \pm SD or number (\%).

NS, not significant.
FSH levels (10.9 vs. 8.2) between the sclerotherapy group and controls. Although the number of oocytes retrieved after stimulation was larger in group 1, the difference was not statistically significant (12.1 vs. 6.1). Two cases of ectopic pregnancy were found in group 1. Patients had a prior history of surgery in 29 out of 56 patients in group 1 , and in 12 out of 41 patients in group 2. Pregnancy after IVF occurred in 23 cycles out of 61 cycles in group 1, with a pregnancy rate of 0.38 , and in 17 cycles out of 43 cycles in group 2 with a pregnancy rate of 0.40 . Although the pregnancy rate was slightly higher in group 2 , the difference was statistically insignificant (Table 1).

Comparing patients that achieved pregnancy and patients that did not in group 1, age, duration of infertility, BMI, day 3 FSH levels, and prior number of IVF cycles revealed no statistical differences: however, the number of oocytes retrieved after stimulation was significantly higher in the pregnant women (16.4 vs. 9.2). None of the patients in group 1 required hospitalization or unwanted surgical intervention after sclerotherapy (Table 2).

\section{Discussion}

As demonstrated in previous studies, transvaginal ultrasound-guided aspiration of hydrosalpinx had the advantages of being simple and less invasive but was associated with a high recurrence rate and not increasing pregnancy rates. However, these disadvantages may be overcome by injection of sclerosing agents into the fallopian tubes after aspiration of the hydrosalpingeal fluid [10-13]. In a study comparing sclerotherapy and untreated controls in patients diagnosed with hydrosalpinx by Jiang et al. [14], the pregnancy rate was significantly higher in the study group. The author emphasized the value of sclerotherapy, hoping this might replace invasive surgeries such as salpingectomy. The great advantage of our study is that we obtained a pregnancy rate similar to that of the salpingectomy groups and also avoided physical stress, severe pain, and hospitalization.

Table 2. Patient's characteristics in group 1

\begin{tabular}{lccc}
\hline Variable & Pregnant & Non pregnant & $p$-value \\
\hline Patients & 23 & 33 & NS \\
Age & $32.5 \pm 3.5$ & $33.2 \pm 4.5$ & NS \\
Duration of infertility $(\mathrm{yr})$ & $2.4 \pm 1.7$ & $3.2 \pm 1.9$ & $\mathrm{NS}$ \\
Body mass index $\left(\mathrm{kg} / \mathrm{m}^{2}\right)$ & $21.0 \pm 2.0$ & $21.8 \pm 2.3$ & $\mathrm{NS}$ \\
Day 3 FSH & $7.7 \pm 3.9$ & $13.2 \pm 21.9$ & $\mathrm{NS}$ \\
IVF history & $1.4 \pm 2.0$ & $2.7 \pm 2.5$ & $\mathrm{NS}$ \\
Oocytes retrieved & $16.4 \pm 14.6$ & $9.2 \pm 7.1$ & 0.016 \\
Previous surgical history & $11 / 23(48 \%)$ & $18 / 33(55 \%)$ & $\mathrm{NS}$ \\
Bilateral hydrosalpinx & $8 / 23(35 \%)$ & $20 / 33(61 \%)$ & $\mathrm{NS}$ \\
Admission after sclerotherapy & $0 / 23(0 \%)$ & $0 / 33(0 \%)$ & $\mathrm{NS}$ \\
\hline
\end{tabular}

Values are presented as mean \pm SD or number (\%).

$\mathrm{NS}$, not significant. 
Tubal factor infertility is one of the most common indications for IVF, and IVF outcomes vary depending on the cause of obstruction. Hydrosalpinx is known to reduce implantation and increase spontaneous abortion in early pregnancy. Several studies reported that salpingectomy prior to IVF can increase pregnancy rates. However, salpingectomy requires hospitalization, general anesthesia, and surgery (either open or laparoscopic), and reduction of blood flow to the ovaries might result in a diminished ovarian response to gonadotropin, and reduced ovarian blood supply. Ultrasound-guided transvaginal aspiration of hydrosalpinx has many advantages over laparoscopic operation under general anesthesia, including that it is simple, effective, less invasive, inexpensive, and does not require hospitalization.

In our study, the number of oocytes retrieved was greater in the sclerotherapy group than the salpingectomy group (12.1 \pm 11 vs. $6.2 \pm 1.0$ ); however, the difference was not statistically significant. The reason for this discrepancy may be due to the small sample size and large variation in the number of oocytes retrieved in both groups.

In the sclerotherapy group, none experienced side effects, hospitalization due to severe pain, or surgery due to serious complications of the intervention. Furthermore, pregnancy was achieved in 17 out of 43 cycles in the salpingectomy group, and in 23 out of 60 cycles in the sclerotherapy group, which was confirmed both by serum hCG levels and ultrasound imaging. The pregnancy rate was similar in the two groups ( 0.40 for salpingectomy group, 0.38 for sclerotherapy group), and the difference was not statistically significant. The risk of ectopic pregnancy in IVF reported by previous studies is estimated at $2.2 \%$ to $8.6 \%$ without treatment of hydrosalpinx [15]. Our study showed a $3.6 \%$ rate of ectopic pregnancy, and this is not significantly different from that of untreated hydrosalpinx.

Twelve out of 41 cases in the salpingectomy group and 29 out of 56 cases in the sclerotherapy group had a past history of surgical operation, and the proportion was larger in the sclerotherapy group. Of the 29 cases in the sclerotherapy group, there were as many as 10 cases of severe endometriosis that required operation twice or more, suggestive of severe adhesion in the abdominal cavity. Pregnancy occurred in four of them, proving the value of sclerotherapy in inoperable or difficult cases with severe adhesion.

Among 56 patients that received sclerotherapy, there were 15 cases of persistent hydrosalpinx after the first trial of sclerotherapy, and these were subjected to repeated sclerotherapy.

Alcohol leakage into the abdominal cavity resulted in severe pain, but the pain resolved naturally within 10 minutes, and drainage of the intra-abdominal alcohol was also effective in pain relief. None of the cases experienced persisting pain, intra-abdominal bleeding requiring drainage, need for surgery, or hospitalization. Aspiration performed after identification of the precise size and location of the hydrosalpinx with ultrasound and careful infusion of alcohol and a scle- rosing agent performed by a highly skilled professional would reduce pain and recurrence.

Hydrosalpinx reduces the pregnancy rate for various reasons, and must be treated before IVF. The treatment of choice in the past was laparoscopic salpingectomy. However, salpingectomy requires hospitalization and general anesthesia, and there have been several reports of reduced blood flow to the ovaries after the operation. In cases of severe intra-abdominal adhesion for various reasons, salpingectomy is difficult either with open surgery or laparoscopy. We investigated the value of sclerotherapy in managing hydrosalpinx. Sclerotherapy resulted in a larger number of oocytes retrieved, and the pregnancy rate was not significantly different from salpingectomy. Ultrasound-guided sclerotherapy of hydrosalpinx would be a useful option for cases in which severe intra-abdominal adhesion is expected.

\section{Conflict of interest}

No potential conflict of interest relevant to this article was reported.

\section{References}

1. Strandell A, Waldenstrom U, Nilsson L, Hamberger L. Hydrosalpinx reduces in-vitro fertilization/embryo transfer pregnancy rates. Hum Reprod 1994;9:861-3.

2. Blazar AS, Hogan JW, Seifer DB, Frishman GN, Wheeler CA, Haning RV. The impact of hydrosalpinx on successful pregnancy in tubal factor infertility treated by in vitro fertilization. Fertil Steril 1997;67:517-20.

3. Aboulghar MA, Mansour RT, Serour Gl. Controversies in the modern management of hydrosalpinx. Hum Reprod Update 1998; 4:882-90.

4. Bildirici I, Bukulmez O, Ensari A, Yarali H, Gurgan T. A prospective evaluation of the effect of salpingectomy on endometrial receptivity in cases of women with communicating hydrosalpinges. Hum Reprod 2001;16:2422-6.

5. Seli E, Kayisli UA, Cakmak H, Bukulmez O, Bildirici I, GuzelogluKayisli O, et al. Removal of hydrosalpinges increases endometrial leukaemia inhibitory factor (LIF) expression at the time of the implantation window. Hum Reprod 2005;20:3012-7.

6. Zeyneloglu HB, Arici A, Olive DL. Adverse effects of hydrosalpinx on pregnancy rates after in vitro fertilization-embryo transfer. Fertil Steril 1998;70:492-9.

7. Camus E, Poncelet C, Goffinet F, Wainer B, Merlet F, Nisand I, et al. Pregnancy rates after in-vitro fertilization in cases of tubal infertility with and without hydrosalpinx: a meta-analysis of published comparative studies. Hum Reprod 1999;14:1243-9.

8. Chan CC, Ng EH, Li CF, Ho PC. Impaired ovarian blood flow and 
reduced antral follicle count following laparoscopic salpingectomy for ectopic pregnancy. Hum Reprod 2003;18:2175-80.

9. Gelbaya TA, Nardo LG, Fitzgerald CT, Horne G, Brison DR, Lieberman BA. Ovarian response to gonadotropins after laparoscopic salpingectomy or the division of fallopian tubes for hydrosalpinges. Fertil Steril 2006;85:1464-8.

10. Kontoravdis A, Makrakis E, Pantos K, Botsis D, Deligeoroglou E, Creatsas $G$. Proximal tubal occlusion and salpingectomy result in similar improvement in in vitro fertilization outcome in patients with hydrosalpinx. Fertil Steril 2006;86:1642-9.

11. Hammadieh N, Coomarasamy A, Ola B, Papaioannou S, Afnan M, Sharif K. Ultrasound-guided hydrosalpinx aspiration during oocyte collection improves pregnancy outcome in IVF: a randomized controlled trial. Hum Reprod 2008;23:1113-7.

12. Nakagawa K, Ohgi S, Nakashima A, Horikawa T, Irahara M, Saito H.
Laparoscopic proximal tubal division can preserve ovarian reserve for infertility patients with hydrosalpinges. J Obstet Gynaecol Res 2008;34:1037-42.

13. Mijatovic V, Veersema S, Emanuel MH, Schats R, Hompes PG. Essure hysteroscopic tubal occlusion device for the treatment of hydrosalpinx prior to in vitro fertilization-embryo transfer in patients with a contraindication for laparoscopy. Fertil Steril 2010; 93:1338-42.

14. Jiang $\mathrm{H}$, Pei $\mathrm{H}$, Zhang WX, Wang XM. A prospective clinical study of interventional ultrasound sclerotherapy on women with hydrosalpinx before in vitro fertilization and embryo transfer. Fertil Steril 2010;94:2854-6.

15. Clayton HB, Schieve LA, Peterson HB, Jamieson DJ, Reynolds MA, Wright VC. Ectopic pregnancy risk with assisted reproductive technology procedures. Obstet Gynecol 2006;107:595-604. 